\title{
Cosmic rays, gamma rays and neutrinos: a survey of 100 years of research
}

\author{
Christian Spiering ${ }^{\mathrm{a}}$ \\ Deutsches Elektronensynchrotron, DESY Zeuthen, Germany
}

Received 29 May 2012

Published online 29 June 2012

(C) EDP Sciences, Springer-Verlag 2012

The year 2012 marks the hundredth anniversary of the detection of cosmic rays by Victor Hess. This discovery opened the first cosmic window beyond optical astronomy, long before radio, infrared, ultraviolet, X-ray and gamma-ray astronomy extended the accessible range of electromagnetic radiation. In commemoration of Hess' groundbreaking measurement, the contributions of this special issue of EPJH follow the remarkable path of cosmic ray science over the last century.

For his discovery, Hess was awarded the 1936 Nobel Prize in Physics. At that time, the investigation of cosmic rays had already opened the field of particle physics. In 1932, the first antiparticle - the positron - was discovered in cosmic rays. Next came the heavy brother of the electron, the muon, discovered in 1936, followed in 1947 by the pion, the first representative of the immense family of mesons. Until the early fifties, cosmic rays remained the main resource for discovering new particles. Only then, particle accelerators took over as the main tool to explore the micro-cosmos.

Effects of the geomagnetic field on cosmic rays revealed that their energies extend to at least $10 \mathrm{GeV}$. In 1938, the discovery of extensive air showers suggested, that the maximum energies must be higher by five orders of magnitude at least. In the 1960s it became clear that some of these particles reach really breathtaking energies - about $10^{20} \mathrm{eV}$. This dwarfs any ground-based accelerator: it corresponds to about 10 million times the beam energy of the Large Hadron Collider.

Already in the 1930s Galactic supernovae were proposed as possible source candidates. Today we believe that cosmic rays with energies up to some $10^{16} \mathrm{eV}$ are accelerated at the shock fronts of Galactic supernova explosions, although we still lack a water-tight confirmation of this hypothesis. However, if this energy range is yet a puzzle, then the $10^{20} \mathrm{eV}$ range is a mystery. Are these particles due to cosmic acceleration in the vicinity of super-massive black holes, or around star crashes releasing gamma ray bursts, or to something totally different? Accounting for these extreme energies is one of the great challenges in astrophysics.

The history of charged cosmic ray research falls in two sub-periods. The first starts with the pre-history of the subject, followed by Hess' discovery and then the subsequent controversies about the nature of cosmic rays - charged particles versus gamma rays. It also covers the dramatic years when cosmic ray physics laid the

a e-mail: christian.spiering@desy.de 
foundation for particle physics. This period is covered in the article of Michael Walter and Arnold Wolfendale [Walter 2012].

The second period addresses two problems.

The one was and is the understanding of sources and acceleration mechanisms at highest energies and the propagation of particles through space. These efforts are based on the detection of extensive air showers. The article of Karl-Heinz Kampert and Alan Watson [Kampert 2012] traces the history of the discovery of air-showers and illustrates how advances in experiment and theory have led to an improved understanding of this phenomenon. It ends with the important data provided by contemporary instruments on the energy spectrum, the mass composition and the arrival direction distribution of high-energy cosmic rays.

The other focus of the second period is the exact measurement of the primary particles. They consist of protons, light and heavy nuclei, with a minuscule admixture of electrons. Precise information on the composition cannot be obtained by air shower studies but only by detecting the primary particles itself, i.e. by exploiting detectors on high-altitude balloons or satellites. These "direct" measurements have revealed much detail about the Galactic cosmic rays below $10^{15} \mathrm{eV}$, where the sensitivity of air shower detector becomes marginal. Balloon and satellite detectors are also unique in their capability to distinguish between primary particles and their antiparticles. Apart from being generated in cosmic ray collisions, antiparticles could have also a more exotic origin, for instance dark matter annihilation, or could be survivors from the Big Bang - both possibilities leading to fundamental consequences. Direct observations of cosmic rays are reviewed in the article of Dietrich Müller [Müller 2012].

The search for the sources of cosmic rays is a three-fold assault, using charged cosmic rays, gamma rays and neutrinos. Cosmic rays are electrically charged, therefore they are deflected by cosmic magnetic fields on their way to Earth. Precise pointing - i.e. astronomy - is only possible with electrically neutral, stable particles: electromagnetic radiation and neutrinos.

First attempts to search for sources of gamma rays with air Cherenkov detectors have been made in the early sixties in Russia. After nearly three decades of stepwise improvements of the instruments - and numerous statistically suspicious "discoveries" of gamma-ray sources -, the first uncontroversial detection of a source of $\mathrm{TeV}$ gamma rays was made in 1989 with the Whipple Telescope in Arizona: the Crab Nebula. Eckard Lorenz and Robert Wagner describe the stony way towards the Crab detection and the impressive success story of Imaging Air Cherenkov Telescopes (IACT) and $\mathrm{TeV}$ gamma-ray astronomy since then [Lorenz 2012].

While nearly 150 sources have been detected by IACTs, the sky-map of extraterrestrial high energy neutrinos is still empty - a challenging terra incognita. Like gamma rays, such neutrinos must be emitted as a by-product of high-energy collisions of charged cosmic rays with matter. But different to gamma rays, neutrinos can provide an uncontroversial proof that their astrophysical sources accelerate hadrons, i.e. the vast majority of cosmic rays.

The first conceptual ideas to detect high energy neutrinos date back to the late fifties. The long evolution towards detectors with a realistic discovery potential started in the seventies and eighties, with the pioneering works in the Pacific Ocean close to Hawaii and in Lake Baikal in Siberia. Today, half a century after the first conceptual ideas, such a detector is in operation: IceCube at the South Pole. The review [Spiering 2012] follows the path from the early fifties to the present time, where we seem to be tantalizingly close to the detection of first extraterrestrial high-energy neutrinos.

The century since Hess' discovery was a remarkable journey. It has provided groundbreaking discoveries in particle physics and has revealed a stunning variety of new cosmic phenomena. This was made possible by ingenious instruments, like sophisticated particle detectors on satellites, Imaging Air Cherenkov Telescopes, huge 
Earth-bound detectors for charged cosmic rays and neutrino detectors deep underground.

Still, we did not yet pinpoint the sources of cosmic rays. But after hundred years of research and an enormous gradient in instrumentation, especially over the last two decades, there is optimism that we will get the first decisive answers very soon.

\section{References}

Kampert, K.H. and A.A. Watson. 2012. Extensive air showers and ultra high-energy cosmic rays: a historial review. Eur. Phys. J. H 37: 359-412

Lorenz, E. and R. Wagner. 2012. Very-high energy gamma-ray astronomy. A 23-year success story in high-energy astroparticle physics. Eur. Phys. J. H 37: 459-513

Müller, D. 2012. Direct observations of galactic cosmic rays. Eur. Phys. J. H 37: 413-458

Spiering, C. 2012. Towards high-energy neutrino astronomy. A historical review. Eur. Phys. J. $H$ 37: 515-565

Walter, M. and A.W. Wolfendale. 2012. Early history of cosmic particle physics. Eur. Phys. J. H 37: 323-358 